\title{
A nomogram to predict the recurrence-free survival and analyze the utility of chemotherapy in stage IB non-small cell lung cancer
}

\author{
Zhenyang Zhang ${ }^{1,2 \#}$, Shuhan Xie ${ }^{1 \#}$, Weijing Cai ${ }^{3 \#}$, Zhi-Nuan Hong ${ }^{1}$, Chuangcai Yang ${ }^{4}$, Yukang Lin ${ }^{5}$, \\ Jiafu Zhu ${ }^{1}$, Zhiwei Lin ${ }^{1}$, Daniel C. Christoph ${ }^{6}$, Hanibal Bohnenberger ${ }^{7}$, Lucyna Kepka ${ }^{8}$, \\ Wolfgang M. Brueckl ${ }^{9,10}$, Paul Van Houtte ${ }^{11}$, Mingqiang Kang ${ }^{1,2 *}$, Jiangbo Lin $^{1,2 *}$
}

${ }^{1}$ Department of Thoracic Surgery, Fujian Medical University Union Hospital, Fuzhou, China; ${ }^{2}$ Key Laboratory of Cardio-Thoracic Surgery (Fujian Medical University), Fujian Province University, Fuzhou, China; ${ }^{3}$ Shanghai Tongshu Biotechnology Co., Ltd., Shanghai, China; ${ }^{4}$ Department of Thoracic and Cardiovascular Surgery, The Second Affiliated Hospital of Fujian Medical University, Fuzhou, China; ${ }^{5}$ Fujian Medical University, Fuzhou, China; ${ }^{6}$ Department of Medical Oncology, Evang. Kliniken Essen-Mitte, Essen, Germany; ${ }^{7}$ Institute of Pathology, University Medical Center, Göttingen, Germany; ${ }^{8}$ Department of Radiotherapy, Military Institute of Medicine, Warsaw, Poland; ${ }^{9}$ Department of Respiratory Medicine, Allergology and Sleep Medicine/Nuernberg Lung Cancer Center, Nuernberg General Hospital, Nuremberg, Germany; ${ }^{10}$ Paracelsus Medical University Nuremberg, Nuremberg, Germany; ${ }^{11}$ Department of Radiation Oncology, Institut Jules Bordet, Université Libre Bruxelles, Brussels, Belgium

Contributions: (I) Conception and design: J Lin, M Kang; (II) Administrative support: M Kang; (III) Provision of study materials or patients: Z Zhang, ZN Hong, S Xie, C Yang; (IV) Collection and assembly of data: ZN Hong, S Xie, C Yang; (V) Data analysis and interpretation: ZN Hong, S Xie, C Yang; (VI) Manuscript writing: All authors; (VII) Final approval of manuscript: All authors.

"These authors contributed equally to this work and should be considered as co-first authors.

*These authors contributed equally to this work and should be considered as co-corresponding authors.

Correspondence to: Mingqiang Kang, MD. Department of Thoracic Surgery, Fujian Medical University Union Hospital, Fuzhou 350001, China. Email: zzycurry24@sina.com; Jiangbo Lin, MD. Department of Thoracic Surgery, Fujian Medical University Union Hospital, Fuzhou 350001, China. Email: jiangbolin8009@sina.com.

Background: Large part of patients of stage IB non-small cell lung cancer (IB NSCLC) may suffer recurrence after surgery. This study is to determine risk factors and establish a nomogram for postoperative recurrence and to provide a reference for adjuvant chemotherapy selection in patients with stage IB NSCLC. Methods: A total of 394 patients with postoperative stage IB NSCLC who visited Fujian Medical University Union Hospital between January 2010 and June 2016 were selected. Patients were divided into training and validation cohorts based on the time of diagnosis. Independent risk factors were identified using a Cox proportional hazards regression model. A nomogram was created to predict recurrence-free survival (RFS) and was validated with an independent cohort. The predictive ability of the nomogram was evaluated using the concordance index (C-index) and calibration curve. RFS between the high- and low-risk groups was determined using Kaplan-Meier curves, and subgroup analysis of chemotherapy was performed.

Results: Visceral pleura invasion, micropapillary structures, tumor size, preoperative serum carcinoembryonic antigen (CEA) level, preoperative serum cytokeratin-19 fragments (Cyfra21-1) level, and postoperative histology were identified as independent risk factors for stage IB NSCLC recurrence. Discrimination of the nomogram showed good prognostic accuracy and clinical applicability, with a C-index of 0.827 and 0.866 in the training and validation cohorts, respectively. The difference in RFS between the high- and low-risk groups in both cohorts was significant $(\mathrm{P}<0.05)$. Finally, a significant difference was observed on whether high-risk group should accept postoperative chemotherapy $(\mathrm{P}<0.05)$.

Conclusions: This nomogram can predict postoperative recurrence probability in patients with stage IB NSCLC, and can select patients with risk factors who need adjuvant chemotherapy.

$\wedge$ ORCID: 0000-0001-5716-6472. 


\begin{abstract}
Keywords: Non-small cell lung cancer (NSCLC); stage IB; adjuvant chemotherapy; nomogram for risk of
\end{abstract} recurrence

Submitted Nov 02, 2021. Accepted for publication Jan 19, 2022.

doi: $10.21037 /$ tlcr-21-1038

View this article at: https://dx.doi.org/10.21037/tlcr-21-1038

\section{Introduction}

At present, lung cancer has the highest rates of morbidity and mortality among all malignancies worldwide (1). According to the histological morphology of cancer cells, lung cancer can be divided into two major categories: non-small cell lung cancer (NSCLC) and small cell lung cancer, with NSCLC accounting for $80-85 \%$ of the cases (2). In recent years, with the development of chest imaging technology, the differentiation of small lung nodules has become increasingly precise, and the detection rate for early-stage lung cancer has been increasing annually (3). Stage I lung cancer patients are generally included in the early-stage lung cancer cohort. Currently, surgery is the main treatment for stage I NSCLC in clinical practice. Previous studies have reported that the recurrence rate of stage I NSCLC patients within 5 years after surgery is $18-29 \%$ (4), with age, sex, pathological (p) and clinical $(\mathrm{T})$ stage status, surgical approach, and tumor size being independent risk factors (5). Considering the high rate of recurrence, it is necessary to identify the relevant risk factors of NSCLC and take the necessary precautions to prevent it. Compared to the $7^{\text {th }}$ edition of the TNM staging system, the stage $\mathrm{T} 2$ in the $8^{\text {th }}$ edition was changed: the maximum diameter of primary tumor is not greater than $5 \mathrm{~cm}$, or has any of the following: the $\mathrm{T}$ stage was determined only by the maximum dimension of the invasive component and excludes the lepidic component and the size range of the T2a was also reduced from 3-5 to 3-4 cm (6). Clearly, patients with stage II and IIIA NSCLC benefit from adjuvant chemotherapy, but this treatment remains controversial for patients with stage IB disease $(7,8)$. According to the National Comprehensive Cancer Network (NCCN) guidelines (version 2, 2021), postoperative adjuvant chemotherapy is recommended for patients with high-risk factors (poorly differentiation, lymphatic-vascular invasion, visceral pleural invasion etc.) (9). Previous studies also show the risk factors of recurrence in IB NSCLC patients are histology, smoking history and LVI (10). In this study, we put more indicators into consideration to predict the recurrence of IB NSCLC, such as elevated preoperative serum carcinoembryonic antigen, which is one of most commonly used marker to evaluate the prognosis of cancer (11).

To establish a quantitative relationship between risk factors and tumor recurrence, survival and risk models can provide an early warning for recurrence or metastasis. To simplify the complex regression equation model, this study transforms the quantitative model into visualized graphs, a nomogram. Based on the nomogram, risk stratification of patients can aid both patients and physicians in making better evaluations regarding recurrence as well as suitable decisions for adjuvant chemotherapy. We present the following article in accordance with the TRIPOD reporting checklist (available at https://tlcr.amegroups.com/article/ view/10.21037/tlcr-21-1038/rc).

\section{Methods}

\section{Study population}

We reviewed our thoracic database and selected patients with stage IB NSCLC from January 2010 to June 2016 at Fujian Union Hospital. A total of 394 patients were included in this retrospective analysis. Patients who received radical treatment between January 2010 and December 2014 were assigned into the training cohort $(n=280)$, while patients from January 2015 to June 2016 were assigned into the validation cohort $(n=114)$ for validation $(12,13)$. The TNM staging system $8^{\text {th }}$ edition was retrospectively used for all of the patients in our study. Patients received adjuvant chemotherapy based on their pathological situation and high-risk factors. The inclusion criteria were as follows: (I) the postoperative pathological type was primary NSCLC with stage IB; (II) patients who did not receive preoperative neoadjuvant treatments such as radiotherapy, immunotherapy, or chemotherapy; and (III) patients who underwent surgical treatment with $\mathrm{R} 0$ resection after the first discovery of a lung mass. The exclusion criteria were as follows: (I) patients with incomplete clinical information; (II) patients with past history of cancer; (III) patients with positive surgical margins (R1 or R2). The diagnostic criteria for recurrence were as follows: (I) local progression or distal metastasis was suggested by postoperative computed 
tomography (CT) scan or positron emission tomography (PET); (II) local or distant puncture for biopsy or second surgery was performed for pathological examination if necessary and possible. Recurrence means local progression or distant metastasis after surgery, whichever occurs first in patients. The study was approved by the Ethics Committee of Fujian Medical University Union Hospital (No. 2022KY006). Individual consent for this retrospective analysis was waived. All procedures performed in this study were in accordance with the Helsinki Declaration (as revised in 2013).

\section{Clinical characteristic and variables}

The basic information of patients included the age, sex, history of smoking, history of hypertension, history of diabetes, pathological variables (including presence of micropapillary component, lymphatic-vascular invasion, etc.), and number of nodes removed. The hematology and laboratory parameters included preoperative platelet to lymphocyte ratio (PLR), preoperative prognostic nutritional index $[\mathrm{PNI}=$ serum albumin $(\mathrm{g} / \mathrm{L})+5 \times$ absolute peripheral blood lymphocyte count $\left.\left(\times 10^{7} / \mathrm{L}\right)\right]$, preoperative peripheral serum tumor marker carcinoembryonic antigen (CEA), and cytokeratin 19 fragment (Cyfra21-1) (14) The platelet count, absolute lymphocyte values, serum albumin levels, CEA, and Cyfra21-1 were obtained from peripheral blood samples taken from patients within 7 days before surgery.

\section{Post-operative follow-ups}

Postoperative follow-ups were started from the date of surgery and performed every 3 months in the first year, every 6 months in the second year, and annually thereafter (totally 5 years) by CT scanning or PET-CT. Recurrencefree survival (RFS) was defined as the time from the date of surgery to the date of recurrence, death from any cause, or censored at the time points of last follow-up. Close followup can detect recurrence at an earlier time.

\section{Statistical analysis}

X-TILE software was used to determine the optimal threshold for PLR, PNI indicators, tumor size, and number of lymph nodes removed. The Kaplan-Meier method was used to analyze the RFS and plot the survival function curves, and the log-rank test was applied to compare the differences in RFS among independent factors. Univariate analysis was used to analyze all single factors affecting RFS, and each statistically significant single factor was included in the multivariate analysis. Based on the independent risk factors, a nomogram was created using the R software.

The performance of the nomogram was evaluated using discrimination and calibration. The model's discrimination was determined by combining two validation methods: the one validation (bootstrap method with 1,000 resamples) to obtain Harrell's concordance index (C-index), and the validation cohort was used to further validate the prediction model. Calibration curves were plotted to calibrate the model. Finally, the high- and low-risk groups were separated based on the total risk score from the nomogram. The Kaplan-Meier method was used to analyze the RFS between the two risk groups as well as the RFS for chemotherapy in the high- and low-risk groups in both cohorts (subgroup analysis). All statistical test levels were considered statistically significant at $\mathrm{P} \leq 0.05$. Statistical analyses were performed using SPSS (version 23.0; SPSS, IBM Corp., Armonk, New York, USA), X-TILE 3.6.1 software (Yale University, New Haven, CT, USA) (15), and R software version 4.0.5 (http:// www.r-project.org). The packages of $\mathrm{R}$ software included rms, rmda, survminer, and survival.

\section{Results}

\section{Study cobort and clinical features}

A total of 394 patients with stage IB NSCLC were eligible for enrollment. In the training cohort, 173 patients were male and 107 were female, among which 36.8\% ( $n=103)$ of patients received adjuvant chemotherapy. In the validation cohort, there were 76 males and 38 females, among which $59.6 \%(n=68)$ of patients received adjuvant chemotherapy (the kinds of chemotherapy are shown in Table S1). The median and mean follow-up times were 60 and 54.7 months, respectively. There were $66 / 394$ (16.7\%) patients who experienced recurrence within 5 years. The distribution of demographics and clinicopathologic characteristics of patients in the two cohorts are shown in Table 1.

\section{Assignment of continuous variables to dichotomous variables}

Continuous variables such as age, tumor size, PLR, PNI, and number of lymph nodes dissected were converted into dichotomous variables by calculating their optimal cutoff values using the X-TILE software. The optimal cutoff values were obtained for each variable as follows: PLR 
Table 1 Demographics and clinicopathologic characteristics of training and validation cohort

\begin{tabular}{|c|c|c|c|}
\hline Characteristics & $\begin{array}{l}\text { Training } \\
\text { cohort }\end{array}$ & $\begin{array}{c}\text { Validation } \\
\text { cohort }\end{array}$ & $P$ value \\
\hline Age (35-80 years) & & & 0.996 \\
\hline$\leq 65$ years & 172 & 70 & \\
\hline$>65$ years & 108 & 44 & \\
\hline Gender & & & 0.362 \\
\hline Male & 173 & 76 & \\
\hline Female & 107 & 38 & \\
\hline Smoking history & & & 0.805 \\
\hline Never smokers & 163 & 66 & \\
\hline Ever smokers & 33 & 16 & \\
\hline Smokers & 84 & 32 & \\
\hline Family history of NSCLC & & & 0.200 \\
\hline No & 273 & 114 & \\
\hline Yes & 7 & 0 & \\
\hline Hypertension & & & 0.954 \\
\hline No & 212 & 86 & \\
\hline Yes & 68 & 28 & \\
\hline Diabetes & & & 0.776 \\
\hline No & 258 & 106 & \\
\hline Yes & 22 & 8 & \\
\hline PLR (=PLT/LY) & & & 0.106 \\
\hline$\leq 114.6$ & 123 & 40 & \\
\hline$>114.6$ & 157 & 74 & \\
\hline PNI (=ALB+5*LY) & & & 0.004 \\
\hline$\leq 50.9$ & 165 & 49 & \\
\hline$>50.9$ & 115 & 65 & \\
\hline CEA (ng/mL) & & & 0.834 \\
\hline Normal & 214 & 86 & \\
\hline Abnormal & 66 & 28 & \\
\hline Cyfra21-1 & & & 0.004 \\
\hline Normal & 214 & 71 & \\
\hline Abnormal & 66 & 43 & \\
\hline Primary site & & & 0.582 \\
\hline Left upper lobe & 75 & 33 & \\
\hline Left lower lobe & 44 & 20 & \\
\hline Right upper lobe & 78 & 35 & \\
\hline
\end{tabular}

Table 1 (continued)
Table 1 (continued)

\begin{tabular}{|c|c|c|c|}
\hline Characteristics & $\begin{array}{l}\text { Training } \\
\text { cohort }\end{array}$ & $\begin{array}{l}\text { Validation } \\
\text { cohort }\end{array}$ & $P$ value \\
\hline Right middle lobe & 23 & 10 & \\
\hline Right lower lobe & 60 & 16 & \\
\hline Surgical approach & & & 0.001 \\
\hline Lung lobectomy & 271 & 100 & \\
\hline $\begin{array}{l}\text { Segmentectomy or wedge } \\
\text { resection }\end{array}$ & 9 & 14 & \\
\hline Histology & & & 0.544 \\
\hline Adenocarcinoma & 231 & 89 & \\
\hline Squamous cell carcinoma & 31 & 17 & \\
\hline Others & 18 & 8 & \\
\hline Tumor size & & & 0.256 \\
\hline$\leq 2.4 \mathrm{~cm}$ & 113 & 39 & \\
\hline$>2.4 \mathrm{~cm}$ & 167 & 75 & \\
\hline VPI & & & 0.062 \\
\hline No & 81 & 44 & \\
\hline Yes & 199 & 70 & \\
\hline Micropapillary structure & & & 0.014 \\
\hline No & 240 & 86 & \\
\hline Yes & 40 & 28 & \\
\hline LVI & & & 0.242 \\
\hline No & 272 & 108 & \\
\hline Yes & 8 & 6 & \\
\hline Invasion of bronchus & & & 0.304 \\
\hline No & 263 & 110 & \\
\hline Yes & 17 & 4 & \\
\hline Number of nodes resected & & & 0.040 \\
\hline$\leq 12$ & 42 & 27 & \\
\hline$>12$ & 238 & 87 & \\
\hline Obstructive pneumonia & & & 0.327 \\
\hline No & 275 & 114 & \\
\hline Yes & 5 & 0 & \\
\hline Adjuvant chemotherapy & & & $<0.001$ \\
\hline No & 177 & 46 & \\
\hline Yes & 103 & 68 & \\
\hline \multicolumn{4}{|c|}{$\begin{array}{l}\text { NSCLC, non-small cell lung cancer; PLR, platelet to lymphocyte } \\
\text { ratio; PLT, platelet; LY, lymphocyte; PNI, prognostic nutritional } \\
\text { index; ALB, albumin; CEA, carcinoembryonic antigen; Cyfra21-1, } \\
\text { cytokeratin-19 fragments; VPI, visceral pleural invasion; LVI, } \\
\text { lymphatic-vascular invasion. }\end{array}$} \\
\hline
\end{tabular}


$=114.6, \mathrm{PNI}=50.9$, tumor size $=2.4 \mathrm{~cm}$, and number of lymph nodes moved $=12$. The cut-off values for age, CEA, and Cyfra21-1 are 65 years, $5.0 \mathrm{ng} / \mathrm{mL}$, and $3.3 \mathrm{ng} / \mathrm{mL}$, respectively. According to the rules of clinical laboratory in our hospital, the range of the norm of CEA and Cyfra21-1 is $0-5.0 \mathrm{ng} / \mathrm{mL}$ and $0-3.3 \mathrm{ng} / \mathrm{mL}$, separately.

\section{Univariate and multivariate survival analysis and Kaplan-Meier RFS curves}

All variables in Table 1 were calculated using the univariate and multivariate Cox proportional hazards regression model, with each factor $(\mathrm{P}<0.05)$ as follows: histology (squamous cell carcinoma: $\mathrm{P}=0.001<0.05$ and others: $\mathrm{P}=0.007<0.05)$, micropapillary component $(\mathrm{P}=0.002<0.05)$, tumor size $(\mathrm{P}<0.001)$, visceral pleura invasion $(\mathrm{P}<0.001)$, Cyfra $21-1$ $(\mathrm{P}=0.008<0.05)$, and CEA $(\mathrm{P}=0.002<0.05)$ (Table 2). The Kaplan-Meier survival curves for each independent risk factor were plotted separately, as shown in Figure 1. The Kaplan-Meier RFS curves for chemotherapy was shown in Figure S1.

\section{Nomogram for recurrence-free survival}

The six independent risk factors were converted into a nomogram, as shown in Figure 2. To verify the degree of discrimination and calibration of the prediction model, validation methods and a calibration curve can be generally adopted. In one validation, the $\mathrm{C}$-index was 0.827 after 1,000 internal self-sampling times (the closer the consistency index was to 1 , the better the model was for individualized differentiation). In other validation, a C-index of 0.866 in the validation cohort showed that the nomogram had good accuracy and application. The slope of the calibration curve was close to the reference line, indicating that the model could better predict the RFS rate of patients (Figure 3). For instance, a patient with IB NSCLC with tumor size of $2.7 \mathrm{~cm}$ (61 points), histology of adenocarcinoma (0 points), involvement of visceral pleura (100 points) and micropapillary structure (37 points) has a sum-point equal to 198, corresponding to predicted 3 - and 5 -year RFS of $73 \%$ and $56 \%$, respectively.

\section{The clinical value of the nomogram for adjuvant chemotherapy in high-risk groups}

The score of each patient was calculated (retaining one decimal) from the nomogram, and patients were divided into high-risk (total scores $>135$ ) and low-risk (total score $\leq 135$ ) groups. A significant difference in RFS between the two groups was observed in the training and validation cohorts $(\mathrm{P}<0.001$ and $\mathrm{P}=0.008$, respectively $)$, as shown in Figure 4. Subgroup survival analysis in the training cohort was performed in the high- and low-risk groups, which showed that the RFS was higher in patients who received chemotherapy than in those who did not receive the treatment in the high-risk group $(\mathrm{P}=0.003)$. However, this difference was not observed in the low-risk group $(\mathrm{P}=0.220)$. In addition, a difference between accepting chemotherapy and not accepting chemotherapy in the high- and lowrisk groups was observed in the validation cohort $(\mathrm{P}=0.029$ in the high-risk group and $\mathrm{P}=0.791$ in low-risk group), as shown in Figure 5.

\section{Discussion}

The tumor nodes metastasis (TNM) staging system for lung cancer is currently used clinically as an important reference for guiding and predicting patient prognosis. However, the prognostic factors incorporated in this system are limited and still cannot accurately predict the prognosis of patients with early-stage NSCLC. In addition, patients with earlystage NSCLC experience local recurrence and distant metastasis due to micrometastasis $(16,17)$. Therefore, there is a need to explore and incorporate more variables to establish an effective model for predicting the prognosis of early-stage NSCLC, in order to improve the prognosis and survival of patients with stage IB NSCLC by early detection, intervention, and scientific treatment plans.

Previous studies have already demonstrated that nomograms can facilitate the stratification of patients into high- and low-risk groups (18). Therefore, we pooled patients into different risk stratification groups to determine the impact of adjuvant chemotherapy in the different risk groups. In our study, the $\mathrm{C}$-index of the training and validation cohorts were better than those reported in previous studies $(12,19)$, indicating a better discrimination of our nomogram. To the best of our knowledge, the postoperative survival and prognosis model in stage IB NSCLC patients in previous studies have mostly reported overall survival (death) as an endpoint, and few laboratory blood tests (albumin, Cyfra21-1, etc.) have been included as variables in these studies $(20,21)$. Compared to other endpoints, RFS may be a better endpoint for the evaluation of adjuvant chemotherapy (22). In our study, we investigated several laboratory variables and histological subtypes for 
Table 2 Selected factors in the training cohort for building the nomogram through univariate and multivariate analyses

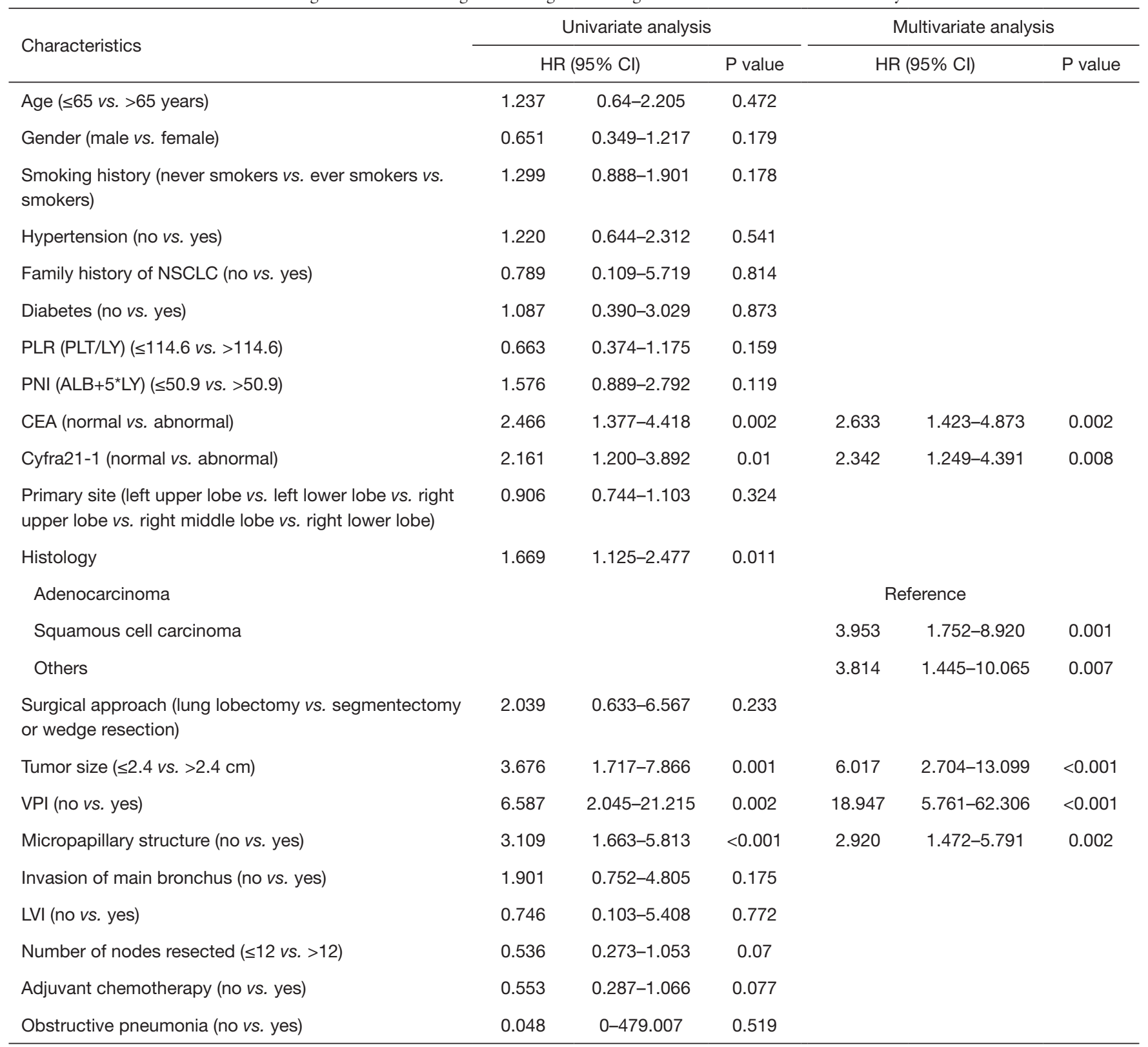

HR, hazard ratio; Cl, confidence interval; NSCLC, non-small cell lung cancer; PLR, platelet to lymphocyte ratio; PLT, platelet; LY, lymphocyte; PNI, prognostic nutritional index; ALB, albumin; CEA, carcinoembryonic antigen; Cyfra21-1, cytokeratin-19 fragments; VPI, visceral pleural invasion; LVI, lymphatic-vascular invasion.

postoperative recurrence in patients with stage IB NSCLC to establish a postoperative recurrence prediction model. This model can be used to screen suitable patients for adjuvant chemotherapy to improve RFS. Moreover, this risk stratification nomogram was used in the validation cohort to validate the feasibility and replicability of the nomogram.

Visceral pleural invasion was an independent risk factor for recurrence in patients with stage IB NSCLC in our series and is now included in the T2 staging criteria of the $8^{\text {th }}$ edition of the international TNM staging system for lung cancer (23). It has been found that there are abundant lymphatic connections between the lung parenchyma and visceral pleura, with drainage converging to the hilar lymph nodes (24). Therefore, patients with NSCLC involving 
A

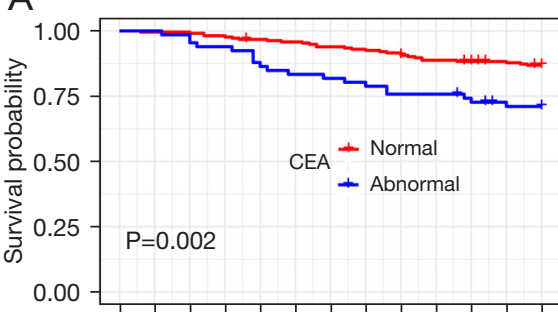

$0 \quad 51015202530354045505560$ Time, month

Number at risk

Normal 214213213210206204200198195188186183179

Abnormal $\quad \begin{array}{llllllllllllll}66 & 66 & 65 & 62 & 58 & 55 & 54 & 53 & 50 & 50 & 48 & 45 & 44\end{array}$

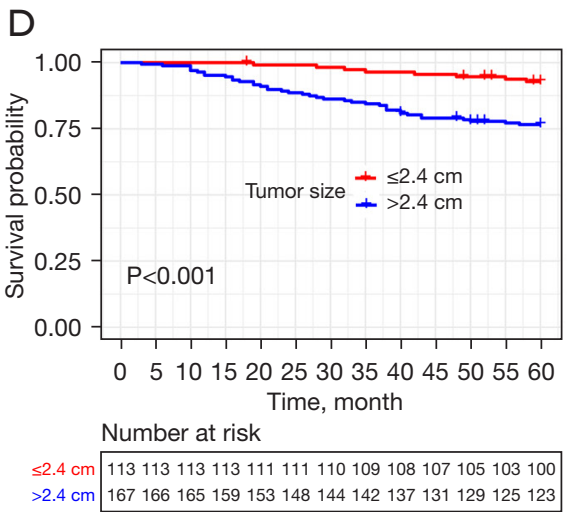

B

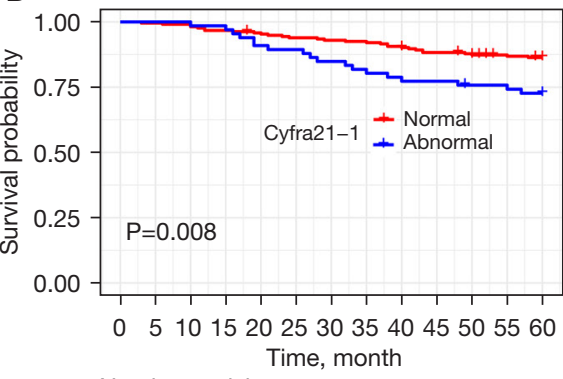

Number at risk

Normal 214213212207204200198197193187185179176

Abnormal $6 \begin{array}{lllllllllllll}66 & 66 & 66 & 65 & 60 & 59 & 56 & 54 & 52 & 51 & 49 & 49 & 47\end{array}$

E

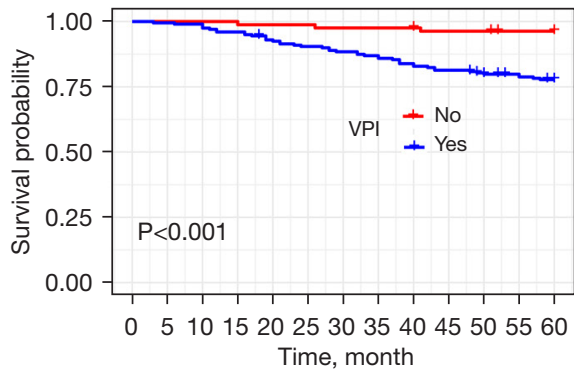

Number at risk

No $\begin{array}{lllllllllllll}81 & 81 & 81 & 81 & 80 & 80 & 79 & 79 & 79 & 77 & 77 & 75 & 75\end{array}$ Yes 199198197191184179175172166161157153148
C

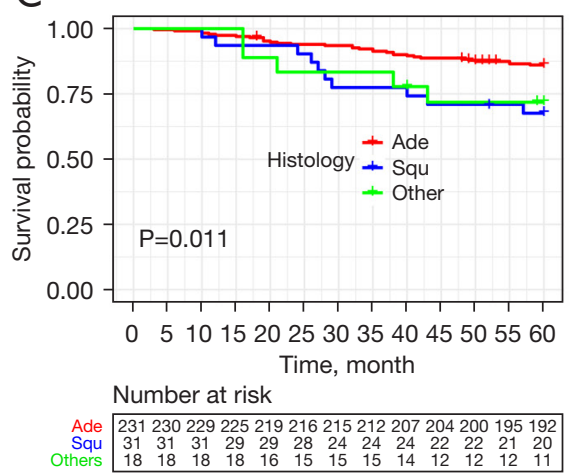

$\mathrm{F}$

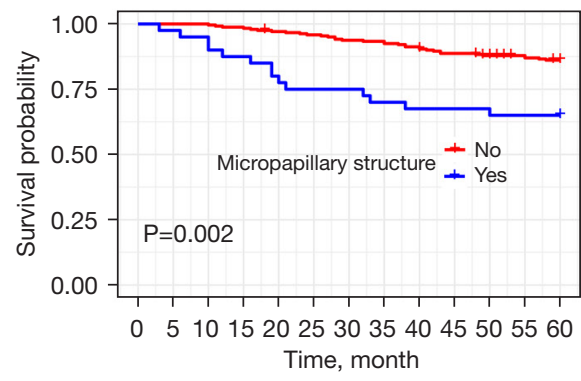

Number at risk

No 240240240237232229224223218211207202197 Yes $\quad \begin{array}{lllllllllllll}40 & 39 & 38 & 35 & 32 & 30 & 30 & 28 & 27 & 27 & 27 & 26 & 26\end{array}$

Figure 1 Kaplan-Meier curves of RFS. Stratified by CEA, Cyfra21-1, histology, tumor size, visceral pleura involvement, micropapillary structure, respectively. RFS, recurrence-free survival; CEA, carcinoembryonic antigen; Cyfra21-1, cytokeratin-19 fragments.

Points
Histology
Micropapillary structure
VPI

Figure 2 Prognostic nomogram for the 3- and 5-year recurrence-free survival. CEA, carcinoembryonic antigen; Cyfra21-1, cytokeratin-19 fragments; VPI, visceral pleura involvement. 
A

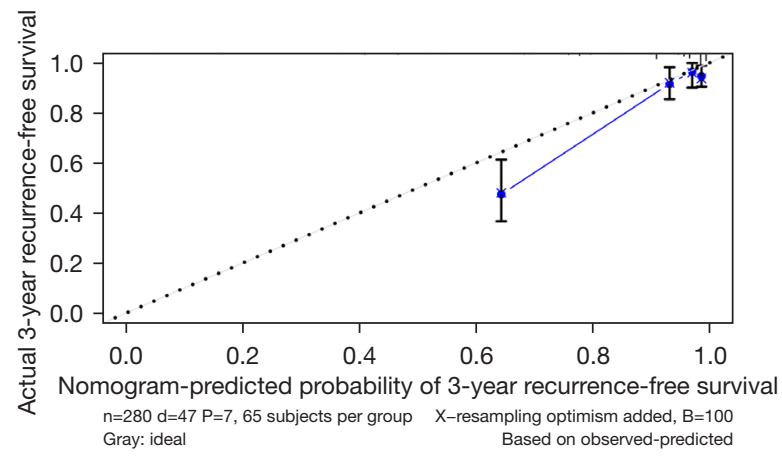

C

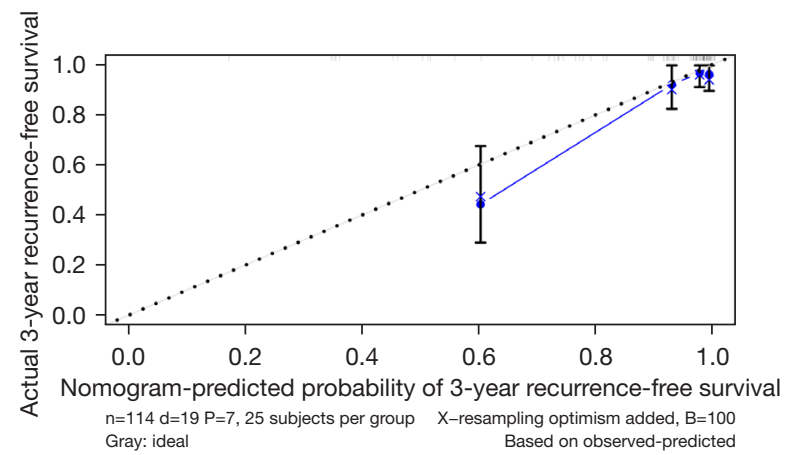

B

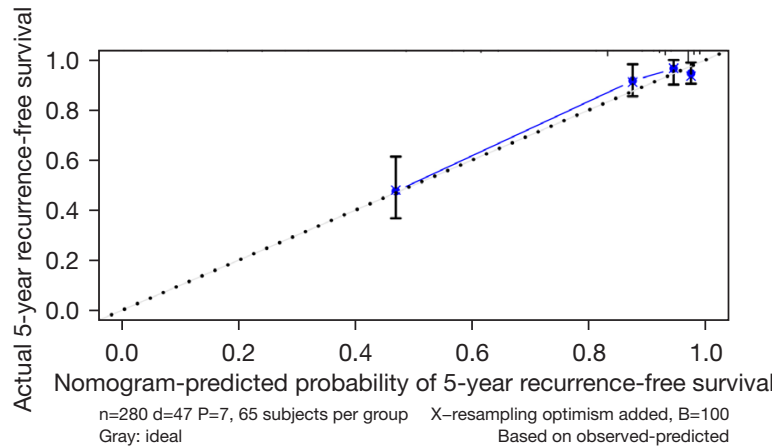

$\mathrm{D}$

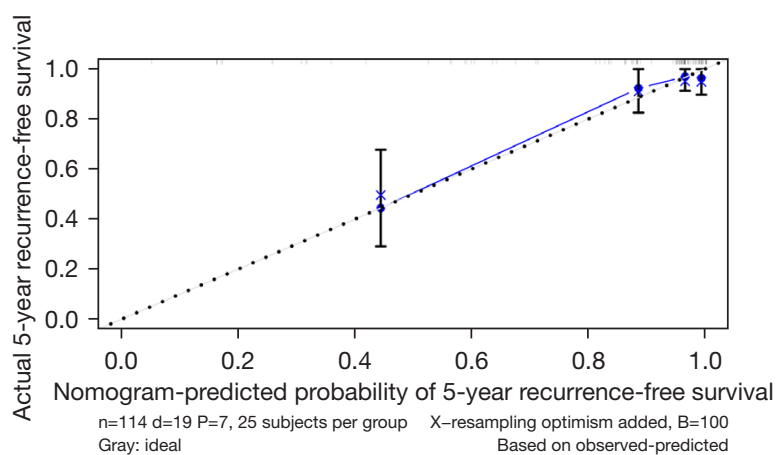

Figure 3 Calibration curves predicting the 3-and 5-year RFS of patients in the training (A,B) and validation (C,D) cohorts. The $\mathrm{x}$-axis indicates the predicted survival probability, and the $\mathrm{y}$-axis indicates the actual survival probability. The 45-degree line indicates that the prediction agrees with actuality. RFS, recurrence-free survival.
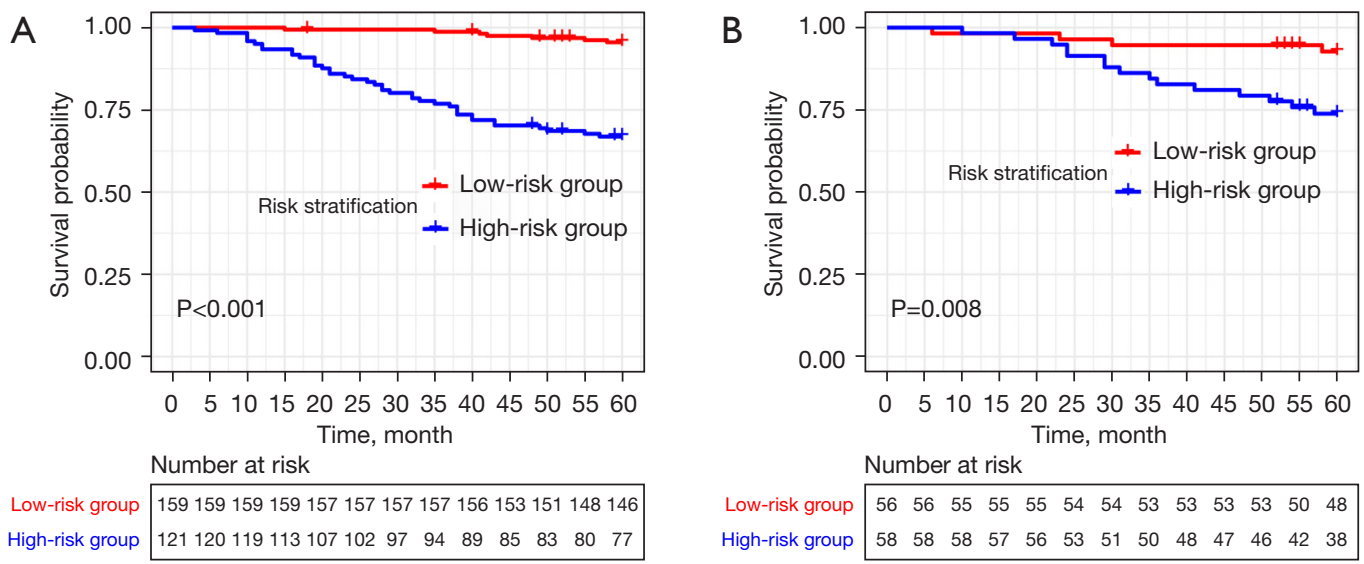

Figure 4 Kaplan-Meier curves of RFS for risk stratification in the training (A) and validation (B) cohorts. RFS, recurrence-free survival.

the visceral pleura have a worse prognosis and are at an increased risk of local recurrence and distant metastasis.

Patients with relatively high preoperative CEA levels have a poorer postoperative prognosis, which is consistent with the results of our study (25). Although CEA levels of patients with long-term smoking habits, cardiovascular disease, gynecological disease, and other diseases are frequently elevated, but the serum CEA level is one of the most commonly used serum tests to evaluate the prognosis of lung cancer $(26,27)$. In addition, studies have shown a better sensitivity of Cyfra21-1 compared to CEA for early stage of lung cancer diagnosis, especially in squamous cell 

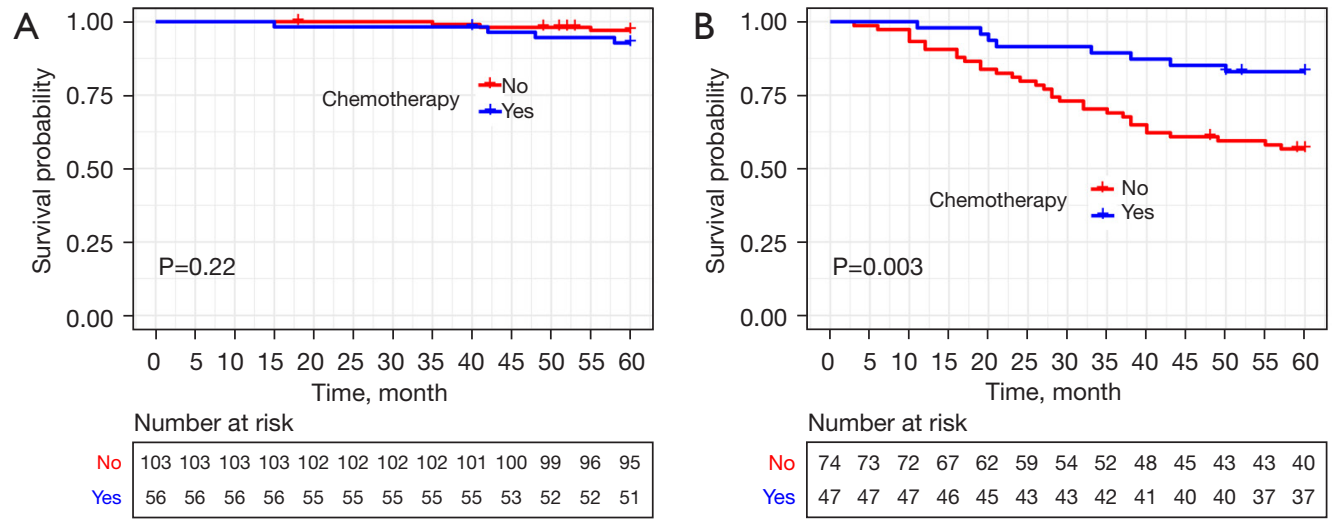

$\begin{array}{llllllllllllll}\text { Yes } & 56 & 56 & 56 & 56 & 55 & 55 & 55 & 55 & 55 & 53 & 52 & 52 & 51\end{array}$

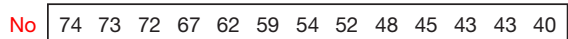

\begin{tabular}{l|lllllllllllll} 
Yes & 47 & 47 & 47 & 46 & 45 & 43 & 43 & 42 & 41 & 40 & 40 & 37 & 37
\end{tabular}
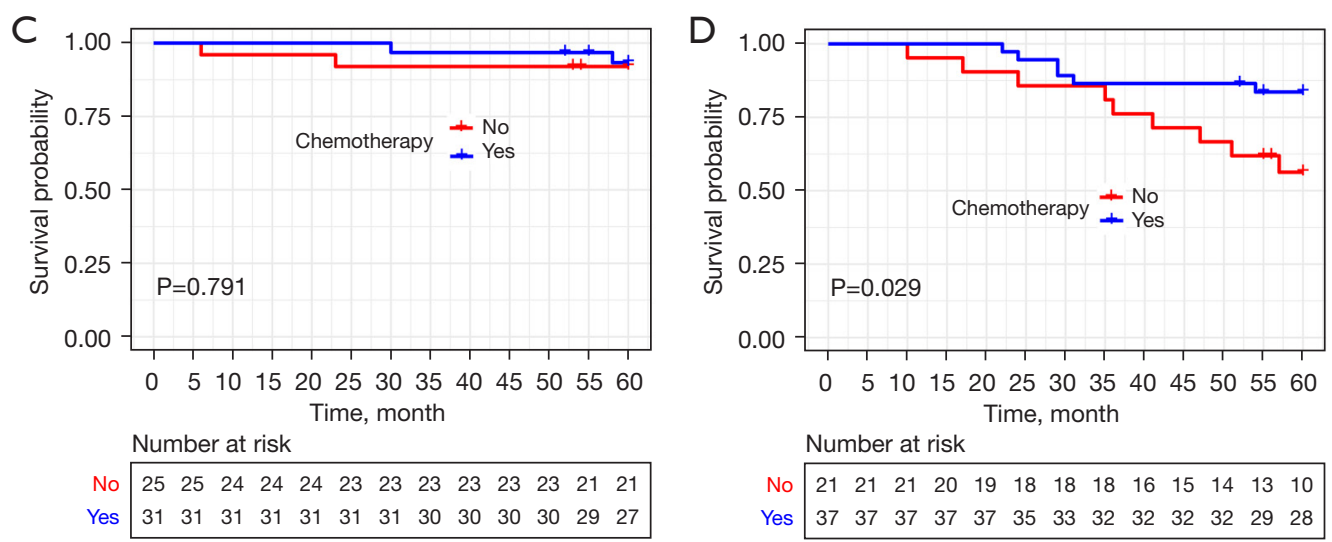

Figure 5 Kaplan-Meier curves of RFS for chemotherapy of the low-risk group in the training cohort (A), high-risk group in the training cohort (B), low-risk group in the validation cohort (C), and high-risk group in the validation cohort (D). RFS, recurrence-free survival.

carcinoma, where the true positive detection rate is much higher than that of CEA and squamous cell carcinoma antigen (SCCA), and is currently considered the most sensitive tumor marker for the diagnosis of NSCLC (28). In 2017, the correlation between Cyfra21-1 levels in the peripheral blood of NSCLC patients and tumor metastasis/ survival status was reported to be stronger than that of CEA and neurospecific enolase (NSE), and showing an independent prognostic effect on patients with different stages of lung cancer (29). The results of this study revealed that these two indicators have sufficient predictive strength in the multivariate analysis of the nomogram.

There is a consensus that adjuvant chemotherapy is not required for patients with stage IA NSCLC resected at R0; however, it is still controversial whether chemotherapy is required for patients with stage IB NSCLC, especially if the tumor involves the visceral pleura (30). According to the National Comprehensive Cancer Network, patients with high-risk factors are recommended to receive adjuvant chemotherapy, but it only determined the possible high- risk factors (8). A previous study also revealed that high-risk patients may benefit from adjuvant chemotherapy (31). In addition, in the subgroup analysis, patients in the low-risk group could choose to refuse chemotherapy after surgery, which does not influence their RFS. However, the subgroup analysis of the high-risk group in the training and validation cohorts suggested that patients in the high-risk group receiving adjuvant chemotherapy had a more significant RFS benefit than those who refused chemotherapy. For local recurrence, salvage surgery can be a good choice, but surgery may be limited or may not be performed owing to poor lung function after the first surgery (32). In addition, although the postoperative pathological reports excluded lymph node metastasis and the postoperative TNM staging was IB stage, micrometastasis may have already occurred, which is difficult to discover and report (33). We recommend that patients with high-risk factors receive chemotherapy after surgery to achieve higher RFS, avoid second surgery for recurrence, and prevent potential metastasis.

A previous study showed that different pathological 
subtypes of lung adenocarcinoma have a significant impact on the prognosis of stage I adenocarcinoma based on the 2015 World Health Organization (WHO) classification (34). In this study, the pathologic type is an independent factor for recurrence, especially the micropapillary structure in lung cancer. It has been shown that patients with NSCLC whose pathological subtype is predominantly micropapillary have a higher risk of recurrence and a worse prognosis $(35,36)$. In particular, for patients with stage I NSCLC, micropapillary structures can be an important prognostic predictor (37). Through Kaplan-Meier and Cox analyses were performed in our study, micropapillary structures were strongly associated with RFS rates. From the nomogram, we can also conclude that the micropapillary structure was one of the most weighted among all the included independent risk factors. Numerous scholars have gradually explored the tumor cell microenvironment and suggested that tumorassociated inflammation contributes to the development of tumor networks (38). However, the results of our study failed to show a connection between inflammation contribution and RFS. A possible explanation for this is that the contribution of inflammation may be more common and obvious in advanced cancers than early-stage cancers.

There are some limitations to our study that should be noted. All cases in this study were from our hospital and represent only a single center and only Asian patients. In the future, it is necessary to further collect data from large samples in different centers, in order to gradually improve the prediction model and provide more accurate postoperative intervention plans for patients with stage IB NSCLC. Besides, there are bias on numbers on chemotherapy between training and validation and the number of use of PET-CT was limited. And it is not clear whether these data and prognostic factors can be used for other ethical groups as well.

\section{Conclusions}

In this study, we found that visceral pleura invasion, micropapillary structures, tumor size, preoperative serum CEA level, preoperative serum CYFRA21-1 level, and histology were independent risk factors for the recurrence of stage IB NSCLC. Our nomogram can aid clinicians in deciding whether patients need adjuvant chemotherapy, which could greatly improve the postoperative RFS of patients. Patients with high-risk factors receiving adjuvant chemotherapy have a more significant RFS benefit. This nomogram can individually predict the probability of postoperative recurrence and guide clinical treatment in patients with stage IB NSCLC.

\section{Acknowledgments}

The authors appreciate the academic support from the AME Lung Cancer Collaborative Group.

Funding: This study was supported by the Fujian Provincial Department of Education (JAT190194); Fujian Medical University Sailing Funding (2019QH1023); Fujian Province Science and Technology Fund Project (2020J01997).

\section{Footnote}

Reporting Checklist: The authors have completed the TRIPOD reporting checklist. Available at https://tlcr. amegroups.com/article/view/10.21037/tlcr-21-1038/rc

Data Sharing Statement: Available at https://tcr.amegroups. com/article/view/10.21037/tlcr-21-1038/dss

Conflicts of Interest: All authors have completed the ICMJE uniform disclosure form (available at https://tlcr.amegroups. com/article/view/10.21037/tlcr-21-1038/coif). WC is from Shanghai Tongshu Biotechnology Co., Ltd., Shanghai, China. DCC reports fees and non-financial support from Amgen, AstraZeneca, Bayer, Boehringer-Ingelheim, BristolMyers Squibb, Chugai, Sharp \& Dohme, Novartis, Pfizer, Roche, Sanofi, Takeda and Boehringer-Ingelheim outside the submitted work. The other authors have no conflicts of interest to declare.

Ethical Statement: The authors are accountable for all aspects of the work in ensuring that questions related to the accuracy or integrity of any part of the work are appropriately investigated and resolved. All procedures performed in this study were in accordance with the Helsinki Declaration (as revised in 2013). The study was approved by the Ethics Committee of Fujian Medical University Union Hospital (No. 2022KY006). Individual consent for this retrospective analysis was waived.

Open Access Statement: This is an Open Access article distributed in accordance with the Creative Commons Attribution-NonCommercial-NoDerivs 4.0 International License (CC BY-NC-ND 4.0), which permits the noncommercial replication and distribution of the article with the strict proviso that no changes or edits are made and the 
original work is properly cited (including links to both the formal publication through the relevant DOI and the license). See: https://creativecommons.org/licenses/by-nc-nd/4.0/.

\section{References}

1. Bray F, Ferlay J, Soerjomataram I, et al. Global cancer statistics 2018: GLOBOCAN estimates of incidence and mortality worldwide for 36 cancers in 185 countries. CA Cancer J Clin 2018;68:394-424. Erratum in: CA Cancer J Clin 2020;70:313.

2. Ju RJ, Cheng L, Xiao Y, et al. PTD modified paclitaxel antiresistant liposomes for treatment of drug-resistant nonsmall cell lung cancer. J Liposome Res 2018;28:236-48.

3. Saghir Z, Dirksen A, Ashraf H, et al. CT screening for lung cancer brings forward early disease. The randomised Danish Lung Cancer Screening Trial: status after five annual screening rounds with low-dose CT. Thorax 2012;67:296-301.

4. Varlotto JM, Recht A, Flickinger JC, et al. Varying recurrence rates and risk factors associated with different definitions of local recurrence in patients with surgically resected, stage I nonsmall cell lung cancer. Cancer 2010;116:2390-400.

5. Koike T, Tsuchiya R, Goya T, et al. Prognostic factors in 3315 completely resected cases of clinical stage I non-small cell lung cancer in Japan. J Thorac Oncol 2007;2:408-13.

6. Moon Y, Choi SY, Park JK, et al. Prognostic factors in stage IB non-small cell lung cancer according to the 8th edition of the TNM staging system after curative resection. J Thorac Dis 2019;11:5352-61.

7. Douillard JY, Rosell R, De Lena M, et al. Adjuvant vinorelbine plus cisplatin versus observation in patients with completely resected stage IB-IIIA non-small-cell lung cancer (Adjuvant Navelbine International Trialist Association [ANITA]): a randomised controlled trial. Lancet Oncol 2006;7:719-27. Erratum in: Lancet Oncol 2006;7:797.

8. Arriagada R, Bergman B, Dunant A, et al. Cisplatinbased adjuvant chemotherapy in patients with completely resected non-small-cell lung cancer. N Engl J Med 2004;350:351-60.

9. National Comprehensive Cancer Network (NCCN). NCCN Clinical Practice Guidelines in Oncology. NonSmall Cell Lung Cancer (Version 2, 2021). Available online: https://www.nccn.org/professionals/physician_gls/ pdf/nsclc.pdf

10. Merritt RE, Abdel-Rasoul M, Fitzgerald M, et al.
Nomograms for Predicting Overall and Recurrence-free Survival From Pathologic Stage IA and IB Lung Cancer After Lobectomy. Clin Lung Cancer 2021;22:e574-83.

11. Kuo SW, Chen JS, Huang PM, et al. Prognostic significance of histologic differentiation, carcinoembryonic antigen value, and lymphovascular invasion in stage I non-small cell lung cancer. J Thorac Cardiovasc Surg 2014;148:1200-7.e3.

12. Churpek MM, Gupta S, Spicer AB, et al. Machine Learning Prediction of Death in Critically Ill Patients With Coronavirus Disease 2019. Crit Care Explor 2021;3:e0515.

13. Xu SJ, Lin GS, Ling HJ, et al. Nomogram to Predict Preoperative Occult Peritoneal Metastasis of Gastrointestinal Stromal Tumors (GIST) Based on Imaging and Inflammatory Indexes. Cancer Manag Res 2020;12:11713-21.

14. Park JW, Chang HJ, Yeo HY, et al. The relationships between systemic cytokine profiles and inflammatory markers in colorectal cancer and the prognostic significance of these parameters. Br J Cancer 2020;123:610-8.

15. Camp RL, Dolled-Filhart M, Rimm DL. X-tile: a new bio-informatics tool for biomarker assessment and outcome-based cut-point optimization. Clin Cancer Res 2004;10:7252-9.

16. Zhang P, Duan J, Bai H, et al. Influence of adjuvant chemotherapy on survival for patients with stage IB and IIA non-small cell lung cancer. Thorac Cancer 2021;12:30-9.

17. Stefani D, Plönes T, Viehof J, et al. Lung Cancer Surgery after Neoadjuvant Immunotherapy. Cancers (Basel) 2021;13:4033.

18. Zhang Y, Sun Y, Xiang J, et al. A clinicopathologic prediction model for postoperative recurrence in stage Ia non-small cell lung cancer. J Thorac Cardiovasc Surg 2014;148:1193-9.

19. Liang W, Zhang L, Jiang G, et al. Development and validation of a nomogram for predicting survival in patients with resected non-small-cell lung cancer. J Clin Oncol 2015;33:861-9.

20. Zuo Z, Zhang G, Song P, et al. Survival Nomogram for Stage IB Non-Small-Cell Lung Cancer Patients, Based on the SEER Database and an External Validation Cohort. Ann Surg Oncol 2021;28:3941-50.

21. Tu Z, Tian T, Chen Q, et al. Overall Survival Analyses following Adjuvant Chemotherapy or Nonadjuvant Chemotherapy in Patients with Stage IB Non-Small-Cell Lung Cancer. J Oncol 2021;2021:8052752. 
22. Kilickap S, Demirci U, Karadurmus N, et al. Endpoints in oncology clinical trials. J BUON 2018;23:1-6.

23. Detterbeck FC, Boffa DJ, Kim AW, et al. The Eighth Edition Lung Cancer Stage Classification. Chest 2017;151:193-203.

24. Kudo Y, Saji H, Shimada Y, et al. Impact of visceral pleural invasion on the survival of patients with non-small cell lung cancer. Lung Cancer 2012;78:153-60.

25. Wang XB, Li J, Han Y. Prognostic significance of preoperative serum carcinoembryonic antigen in nonsmall cell lung cancer: a meta-analysis. Tumour Biol 2014;35:10105-10.

26. Xie HL, Gong YZ, Kuang JA, et al. The prognostic value of the postoperative serum CEA levels/preoperative serum CEA levels ratio in colorectal cancer patients with high preoperative serum CEA levels. Cancer Manag Res 2019;11:7499-511.

27. Yajima S, Nakanishi Y, Matsumoto S, et al. Prognostic significance of the postoperative/preoperative serum CEA level ratio in patients with solitary adrenal metastasis from lung cancer. Mol Clin Oncol 2022;16:10.

28. Stieber P, Hasholzner U, Bodenmüller H, et al. CYFRA 211. A new marker in lung cancer. Cancer 1993;72:707-13.

29. Zhang L, Liu D, Li L, et al. The important role of circulating CYFRA21-1 in metastasis diagnosis and prognostic value compared with carcinoembryonic antigen and neuron-specific enolase in lung cancer patients. BMC Cancer 2017;17:96.

30. Morgensztern D, Du L, Waqar SN, et al. Adjuvant Chemotherapy for Patients with T2N0M0 NSCLC. J Thorac Oncol 2016;11:1729-35.

31. Choi J, Oh JY, Lee YS, et al. Clinical Efficacy of Adjuvant Chemotherapy in Stage IB $(<4 \mathrm{~cm})$ Non-Small Cell Lung Cancer Patients with High-Risk Factors. Korean J Intern Med 2022;37:127-36.

32. Suehs CM, Solovei L, Hireche K, et al. Complication and lung function impairment prediction using perfusion

Cite this article as: Zhang Z, Xie S, Cai W, Hong ZN, Yang C, Lin Y, Zhu J, Lin Z, Christoph DC, Bohnenberger H, Kepka L, Brueckl WM, Van Houtte P, Kang M, Lin J. A nomogram to predict the recurrence-free survival and analyze the utility of chemotherapy in stage IB non-small cell lung cancer. Transl Lung Cancer Res 2022;11(1):75-86. doi: 10.21037/tlcr-211038 and computed tomography air trapping (CLIPPCAIR): protocol for the development and validation of a novel multivariable model for the prediction of post-resection lung function. Ann Transl Med 2021;9:1092.

33. Hu S, Luo M, Li Y. Machine Learning for the Prediction of Lymph Nodes Micrometastasis in Patients with NonSmall Cell Lung Cancer: A Comparative Analysis of Two Practical Prediction Models for Gross Target Volume Delineation. Cancer Manag Res 2021;13:4811-20.

34. Tsuta K, Kawago M, Inoue E, et al. The utility of the proposed IASLC/ATS/ERS lung adenocarcinoma subtypes for disease prognosis and correlation of driver gene alterations. Lung Cancer 2013;81:371-6.

35. Hung JJ, Jeng WJ, Chou TY, et al. Prognostic value of the new International Association for the Study of Lung Cancer/American Thoracic Society/European Respiratory Society lung adenocarcinoma classification on death and recurrence in completely resected stage I lung adenocarcinoma. Ann Surg 2013;258:1079-86.

36. Kris MG, Gaspar LE, Chaft JE, et al. Adjuvant Systemic Therapy and Adjuvant Radiation Therapy for Stage I to IIIA Completely Resected Non-Small-Cell Lung Cancers: American Society of Clinical Oncology/Cancer Care Ontario Clinical Practice Guideline Update. J Clin Oncol 2017;35:2960-74.

37. Tsubokawa N, Mimae T, Sasada S, et al. Negative prognostic influence of micropapillary pattern in stage IA lung adenocarcinoma. Eur J Cardiothorac Surg 2016;49:293-9.

38. Hyder J, Boggs DH, Hanna A, et al. Changes in neutrophil-to-lymphocyte and platelet-to-lymphocyte ratios during chemoradiation predict for survival and pathologic complete response in trimodality esophageal cancer patients. J Gastrointest Oncol 2016;7:189-95.

(English Language Editor: A. Kassem) 
Supplementary

Table S1 Different regimens of chemotherapy in training cohort and validation

\begin{tabular}{lcc}
\hline Regimen of chemotherapy & Training cohort & Validation cohort \\
\hline Docetaxel + platinum-based drugs & 16 & 5 \\
Gemcitabine + platinum-based drugs & 24 & 5 \\
Gemcitabine & 0 & 2 \\
Pemetrexed & 2 & 3 \\
Pemetrexed+ platinum-based drugs & 17 & 43 \\
Etoposide + platinum-based drugs & 0 & 1 \\
Vinorelbine + platinum-based drugs & 5 & 0 \\
Paclitaxel + platinum-based drugs & 39 & 9 \\
\hline
\end{tabular}

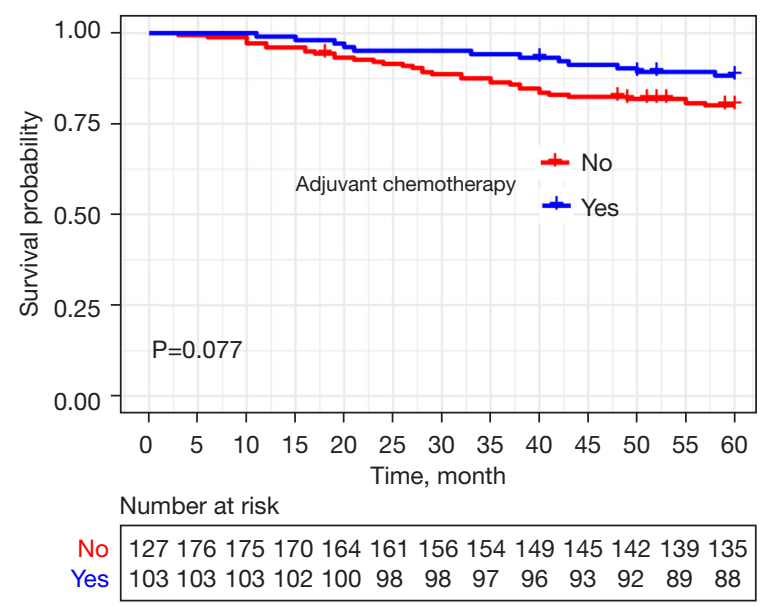

Figure S1 Kaplan-Meier curves for recurrence-free survival (RFS) in regard to the use of adjuvant chemotherapy in the training cohort. 